\title{
Diffraction imaging to understand the internal fabric of mass-transport complexes from Gulf of Cadiz, south west Iberian Margin
}

Jonathan Ford (jford@inogs.it) ${ }^{1,3}$ Roger Urgeles² $\quad$ Eulàlia Gràcia ${ }^{2} \quad$ Angelo Camerlenghi

${ }^{1}$ Istituto Nazionale di Oceanografia e Geofisica Sperimentale (OGS)

${ }^{2}$ Institut de Ciències del Mar (ICM-CSIC)

${ }^{3}$ University of Trieste 


\section{Model of the seismic wavefield}
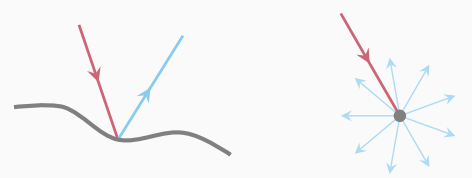

$\underset{\text { reflection image }}{\text { Conventional seismic }}=\underset{\text { Specular }}{\text { refions }}+\underset{\text { energy }}{\text { Diffracted }}+$ Noise

Before migration:

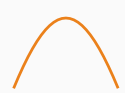

mans

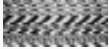
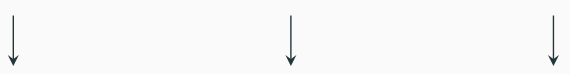

After migration:

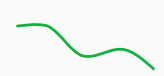

Frosess

37395 


\section{Mass-transport complexes as diffraction generators?}

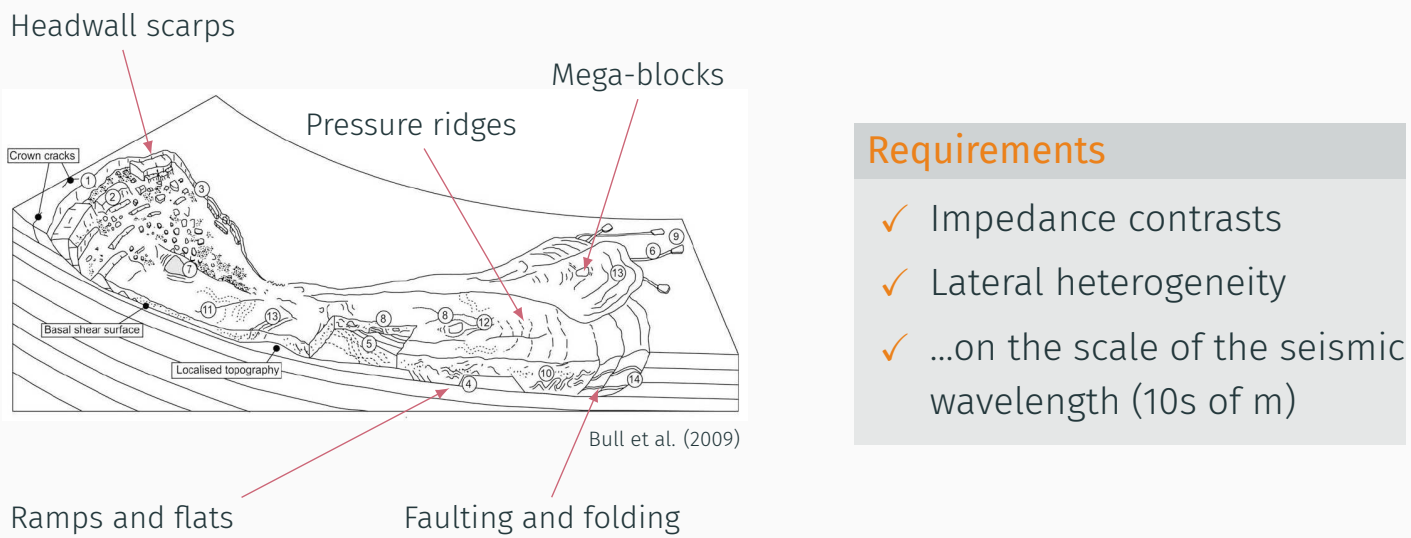




\section{Mass-transport complexes as diffraction generators?}

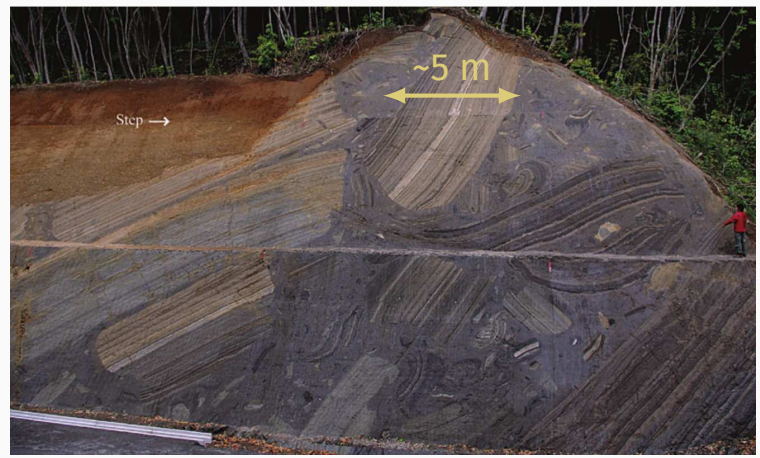

\section{Requirements}

$\checkmark$ Impedance contrasts

$\checkmark$ Lateral heterogeneity

$\checkmark \quad$...on the scale of the seismic wavelength (10s of $\mathrm{m}$ )

Yamamoto et al. (2009) 


\section{Objectives}

Apply diffraction separation and imaging to seismic images of mass-transport complexes:

1. Do MTCs generate significant diffraction energy?

2. Can we use this to image internal structure?

3. Do we resolve things we cannot see in conventional images?

Ford, J., Urgeles, R., Gràcia, E., Camerlenghi, A. (in prep). Diffraction imaging to understand the internal fabric of mass-transport complexes: examples from Gulf of Cadiz, south west Iberian Margin 


\section{Diffraction imaging}

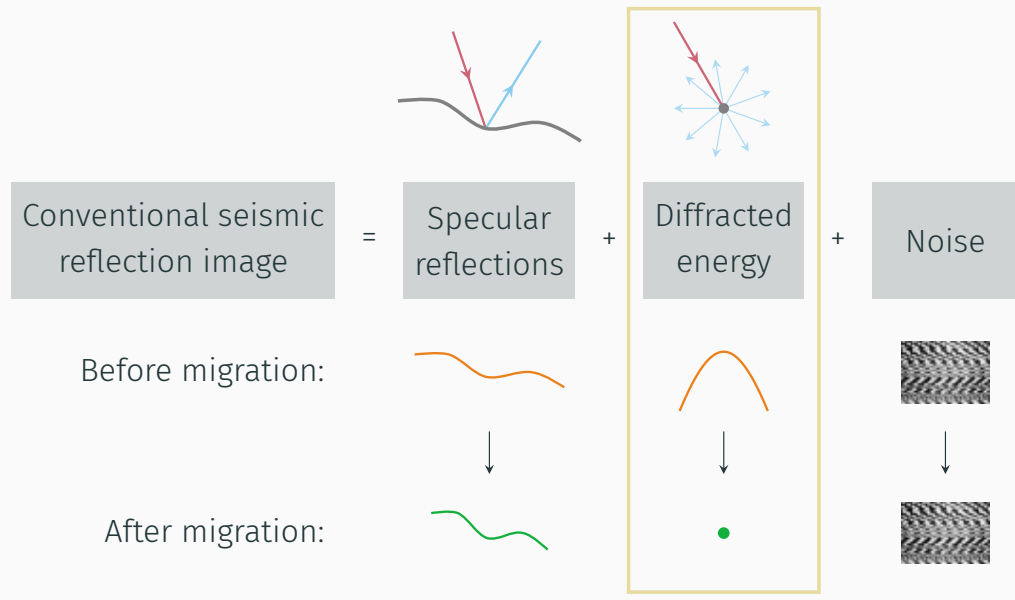

Principle: separate reflected and diffracted wavefields, migrate the diffractions (3) (-) hly to form a diffraction image 


\section{INSIGHT cruises (May 2018 and October 2019)}

\section{INSIGHT}

Imaging large seismogenic and tsunamigenic structures of the Gulf of Cadiz with ultra-high resolution technologies

- PIs: Roger Urgeles and Eulàlia Gràcia

- Acquisition: “2-D HR", 350 m streamer, 930 cu. in. airgun array

\section{ICFMn}

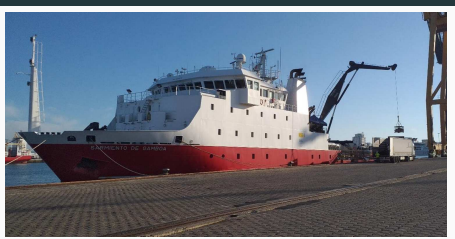

B/O Sarmiento de Gamboa

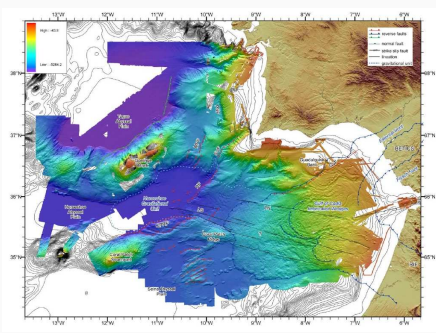

Gulf of Cadiz (Urgeles, 2019) 


\section{Diffraction separation and imaging (normal fault example)}

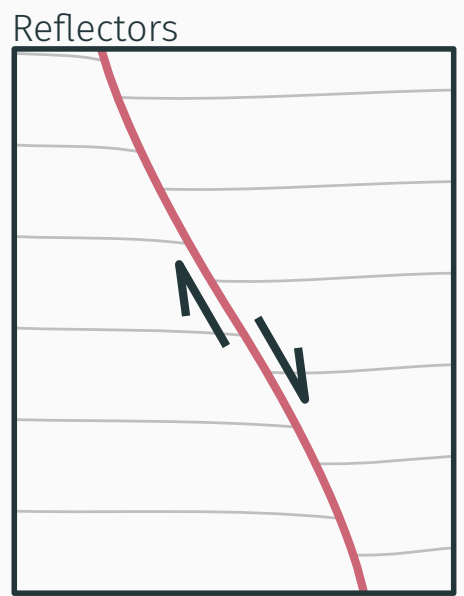

Diffractors

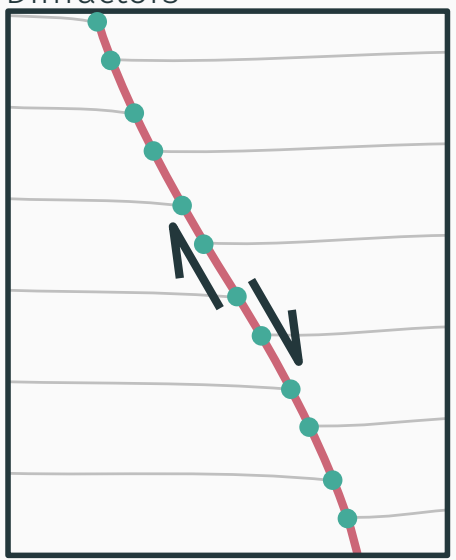




\section{Diffraction separation and imaging (normal fault example)}

- Conventional pre-stack time migration

- Dip estimatation

- De-migrate dip (Ford et al., in prep)

- Diffraction separation (offset domain plane-wave destruction; Fomel et al., 2007)

- Pre-stack time migration of diffractions

Conventional PSTM (INS2-Line1)

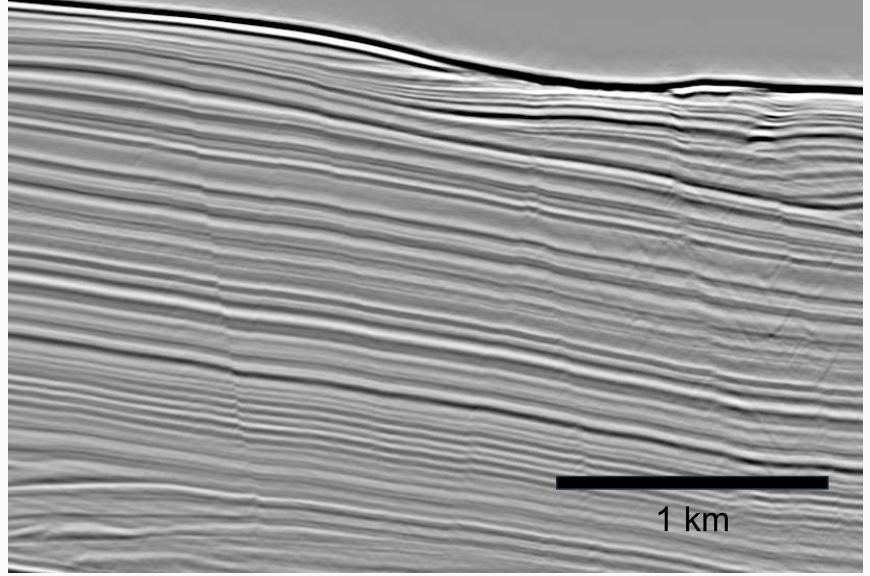




\section{Diffraction separation and imaging (normal fault example)}

- Conventional pre-stack time migration

- Dip estimatation

- De-migrate dip (Ford et al., in prep)

- Diffraction separation (offset domain plane-wave destruction; Fomel et al., 2007)

- Pre-stack time migration of diffractions

Conventional PSTM (INS2-Line1)

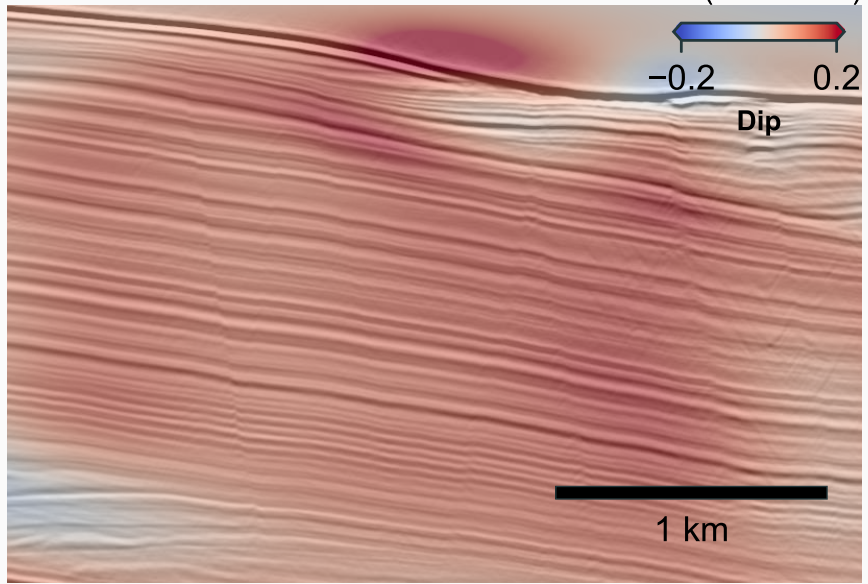




\section{Diffraction separation and imaging (normal fault example)}

- Conventional pre-stack time migration

- Dip estimatation

- De-migrate dip (Ford et al., in prep)

- Diffraction separation (offset domain plane-wave destruction; Fomel et al., 2007)

- Pre-stack time migration of diffractions

Unmigrated image (INS2-Line1)

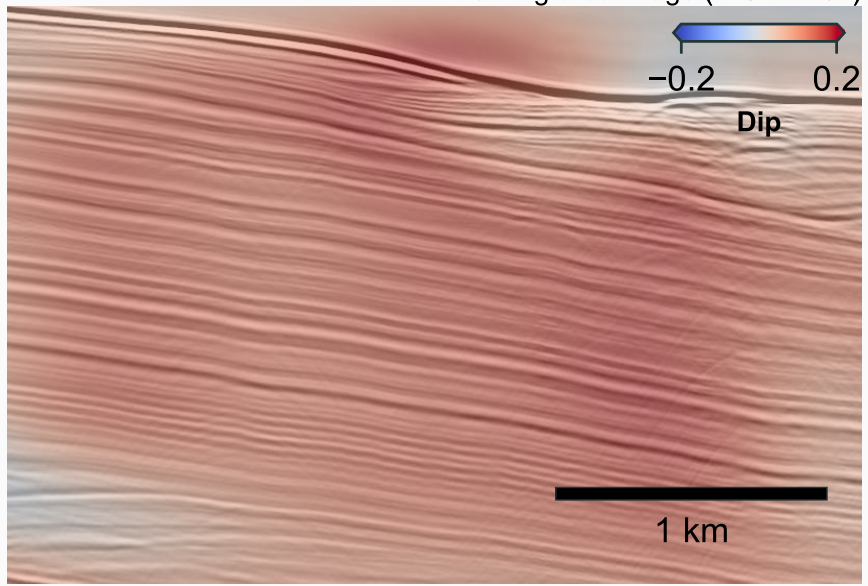




\section{Diffraction separation and imaging (normal fault example)}

- Conventional pre-stack time migration

- Dip estimatation

- De-migrate dip (Ford et al., in prep)

- Diffraction separation (offset domain plane-wave destruction; Fomel et al., 2007)

- Pre-stack time migration of diffractions

Unmigrated image (INS2-Line1)

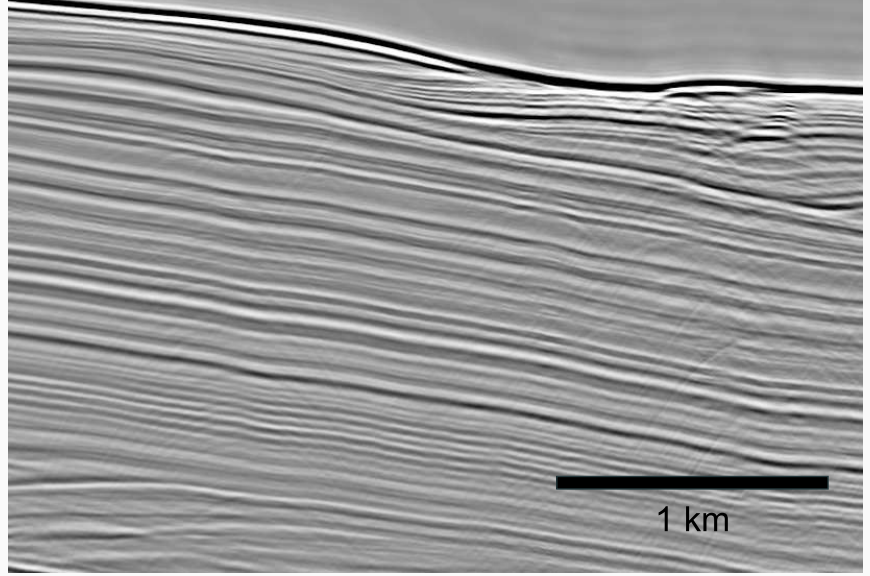




\section{Diffraction separation and imaging (normal fault example)}

- Conventional pre-stack time migration

- Dip estimatation

- De-migrate dip (Ford et al., in prep)

- Diffraction separation (offset domain plane-wave destruction; Fomel et al., 2007)

- Pre-stack time migration of diffractions

Separated diffractions (INS2-Line1)

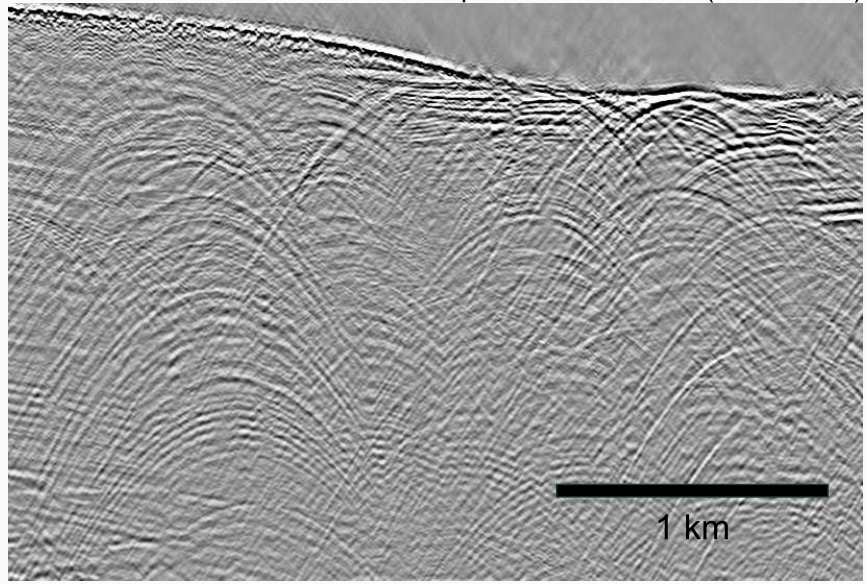




\section{Diffraction separation and imaging (normal fault example)}

- Conventional pre-stack time migration

- Dip estimatation

- De-migrate dip (Ford et al., in prep)

- Diffraction separation (offset domain plane-wave destruction; Fomel et al., 2007)

- Pre-stack time migration of diffractions

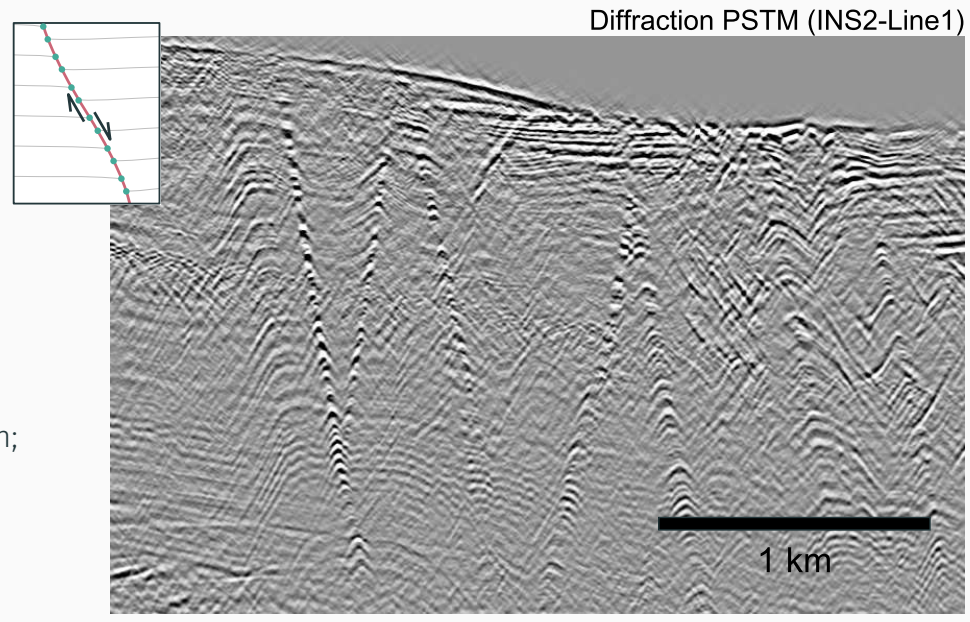




\section{Diffraction separation and imaging (normal fault example)}

- Conventional pre-stack time migration

- Dip estimatation

- De-migrate dip (Ford et al., in prep)

- Diffraction separation (offset domain plane-wave destruction; Fomel et al., 2007)

- Pre-stack time migration of diffractions

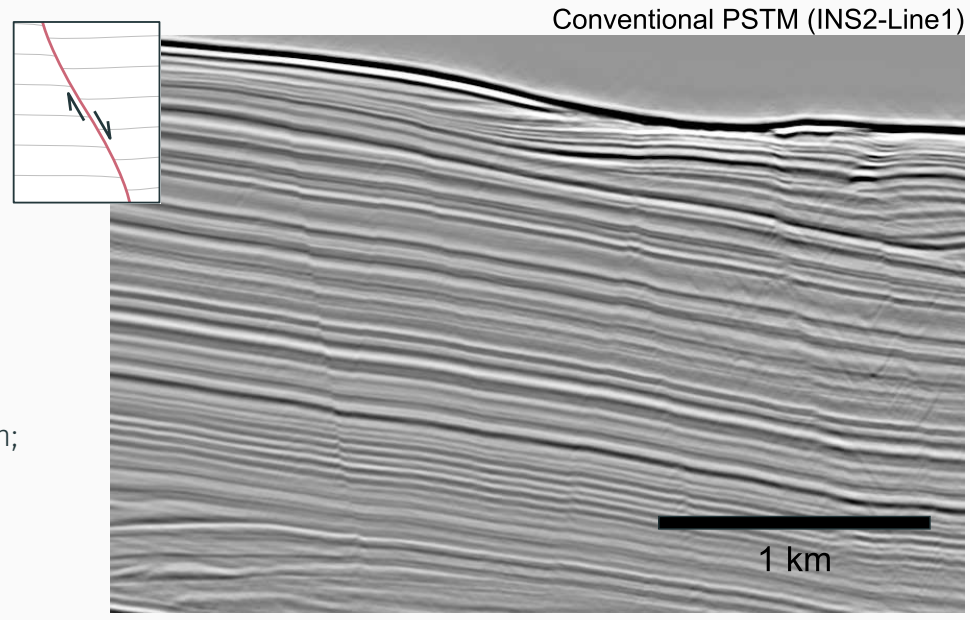




\section{Diffraction separation and imaging (normal fault example)}

- Conventional pre-stack time migration

- Dip estimatation

- De-migrate dip (Ford et al., in prep)

- Diffraction separation (offset domain plane-wave destruction; Fomel et al., 2007)

- Pre-stack time migration of diffractions

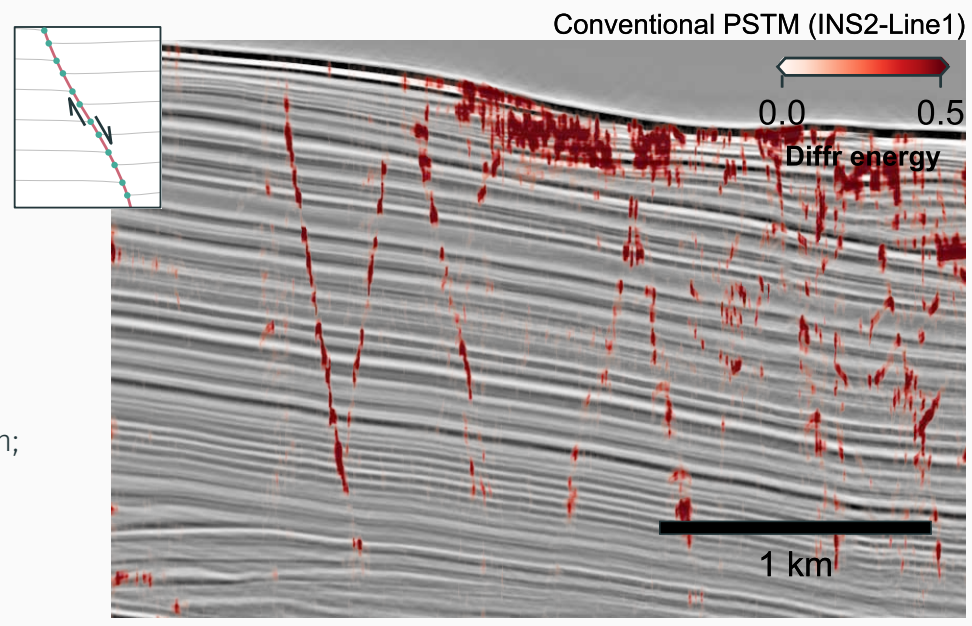




\section{Portimao Bank - Lolita salt diapir}
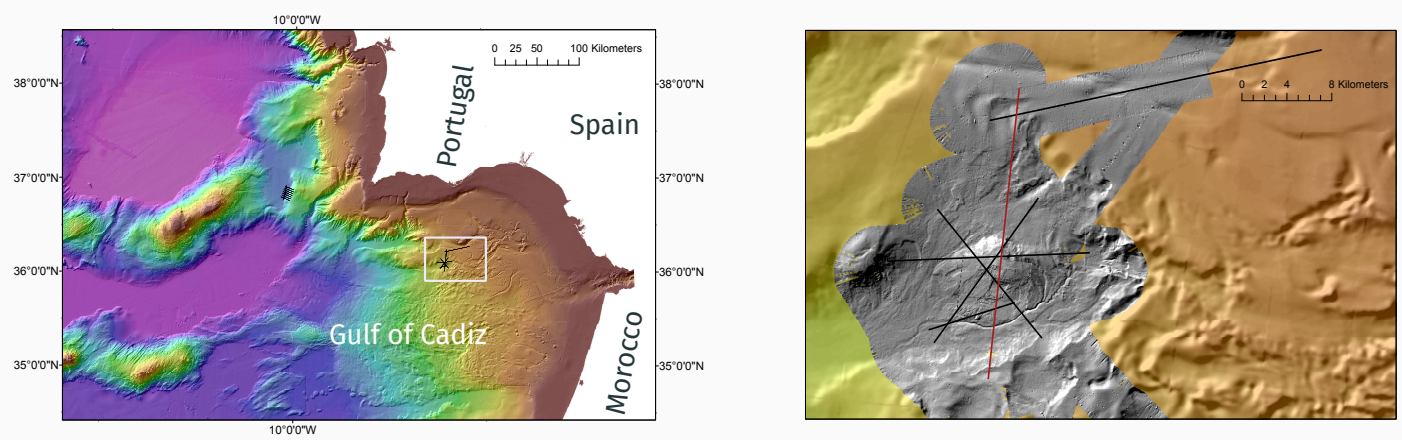

Geological setting: extensive contouritic deposits (from Mediterranean Outflow Water), salt diapirism = mass-wasting 


\section{Portimao Bank - Lolita salt diapir}

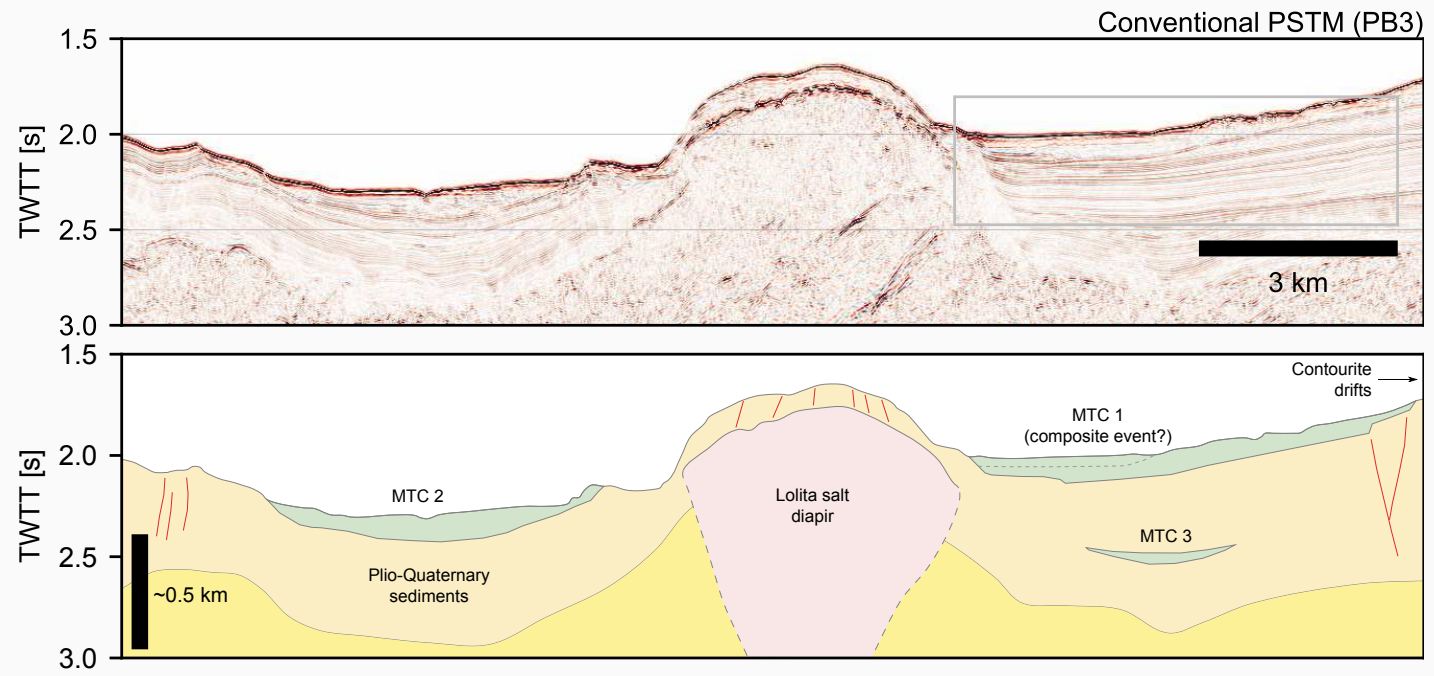




\section{Portimao Bank - Lolita salt diapir}

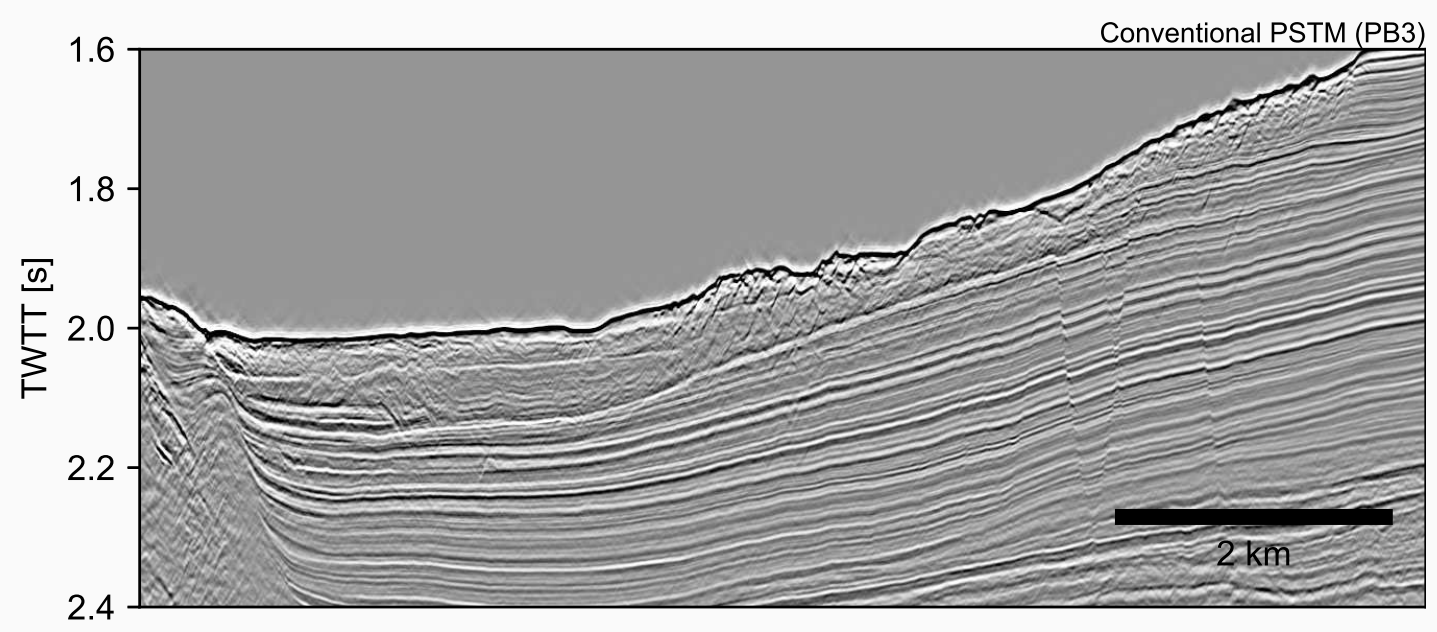




\section{Portimao Bank - Lolita salt diapir}

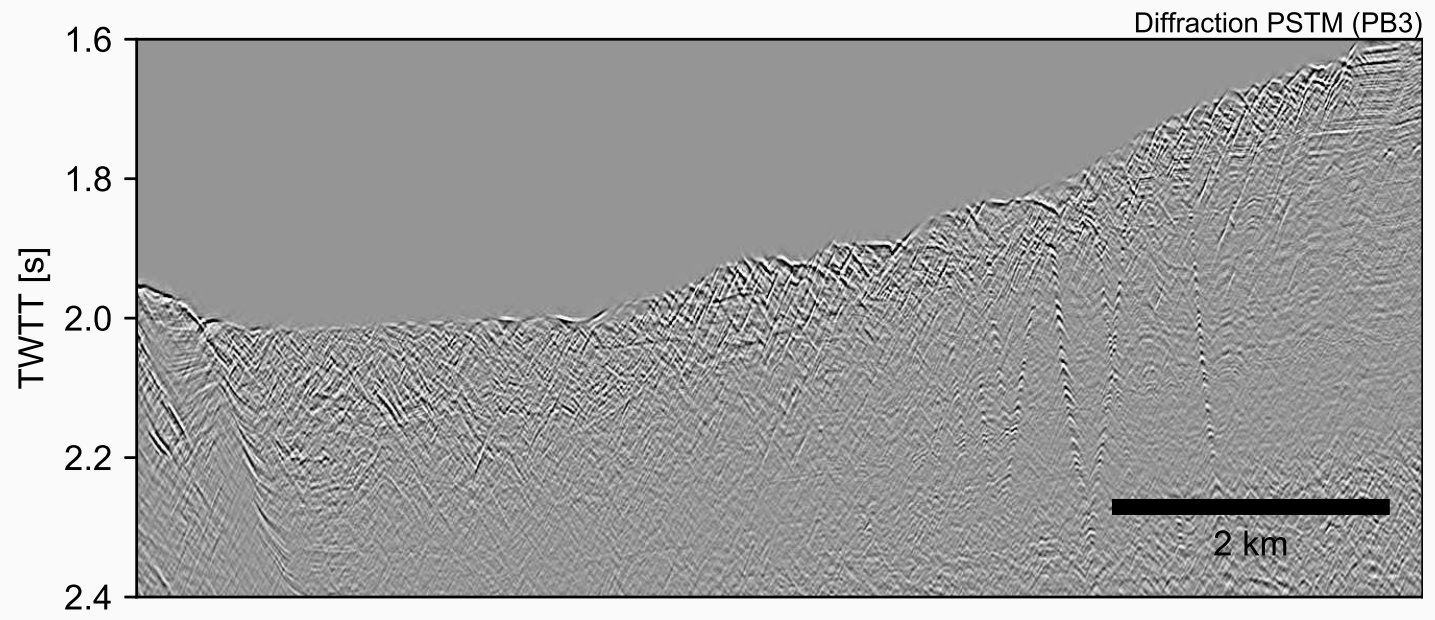




\section{Portimao Bank - Lolita salt diapir}

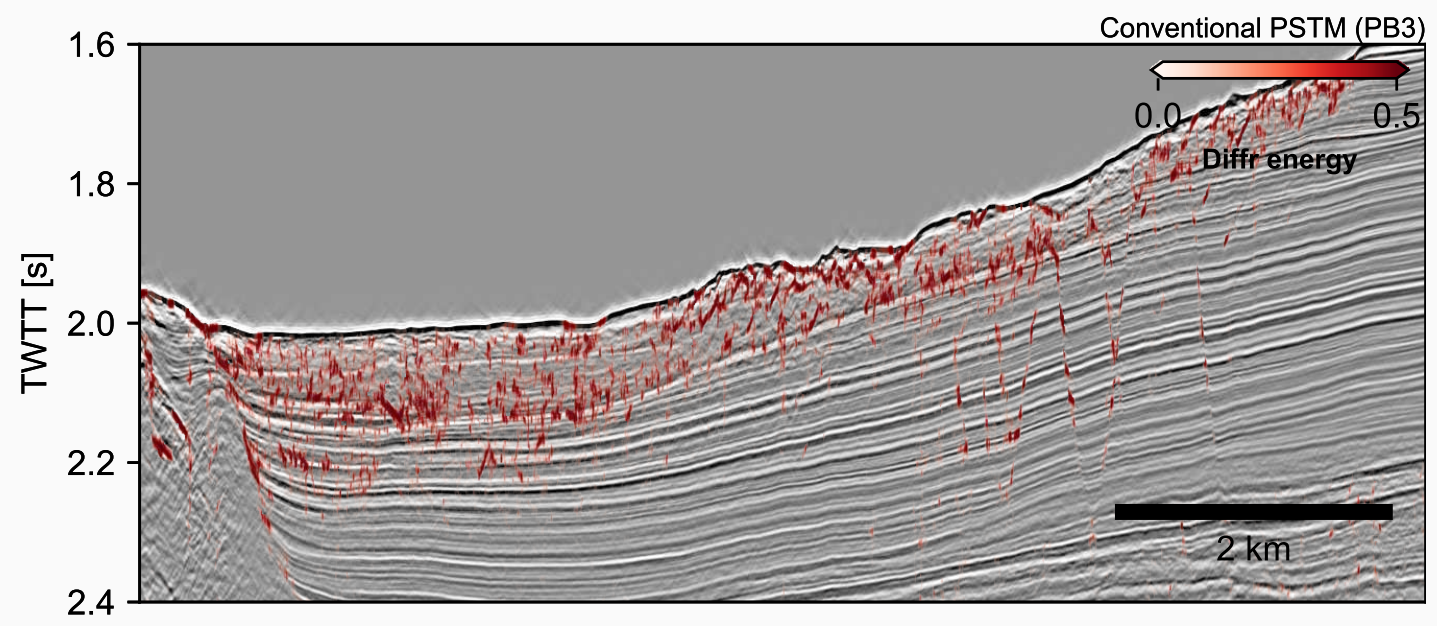




\section{Portimao Bank - Lolita salt diapir}

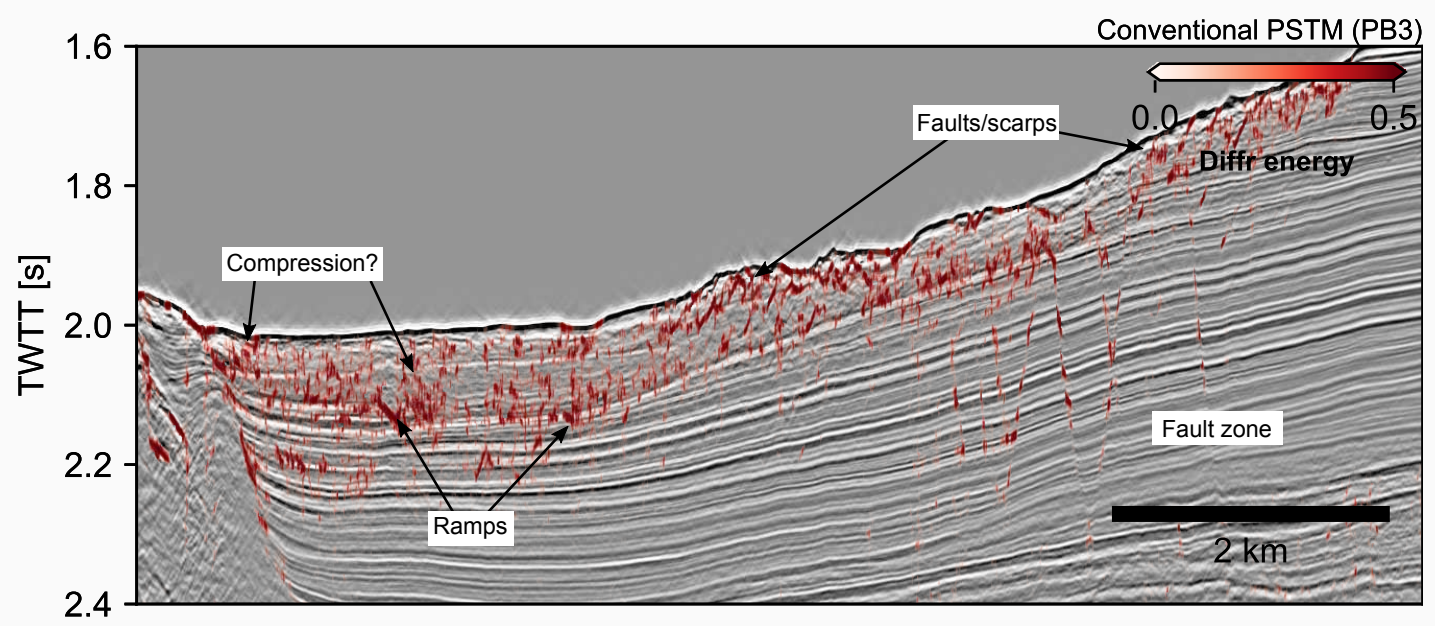




\section{Marques de Pombal Fault}
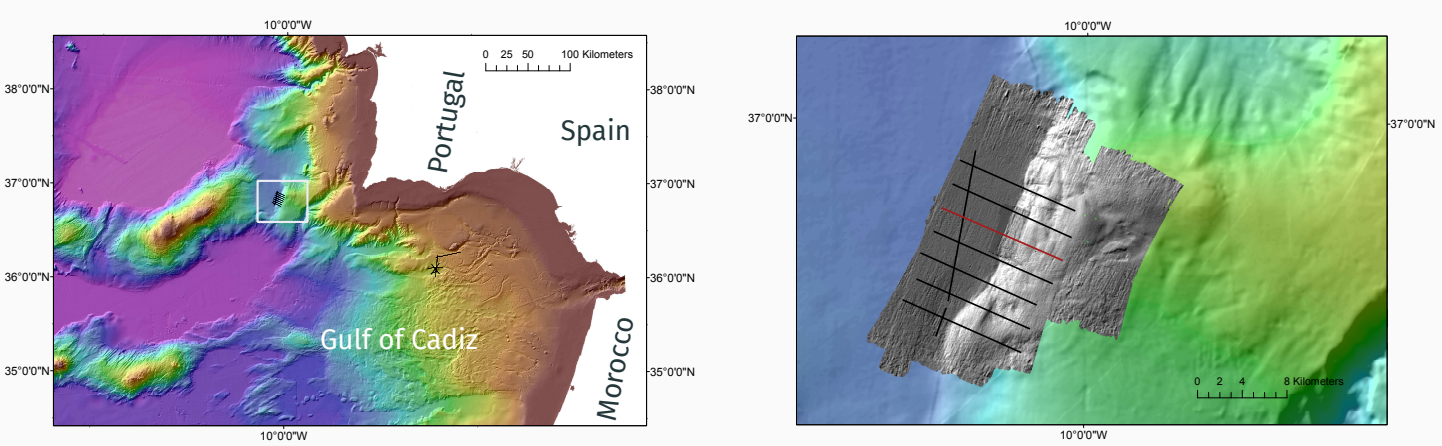

\section{Geological setting:}

- Monoclinal thrust cutting the Plio-Quaternary (potential source of 1755 Lisbon earthquake + submarine landslide + tsunami; Zitellini et al., 2001)

- Thick succession of MTCs in basin - record fault activity? 


\section{Marques de Pombal Fault}
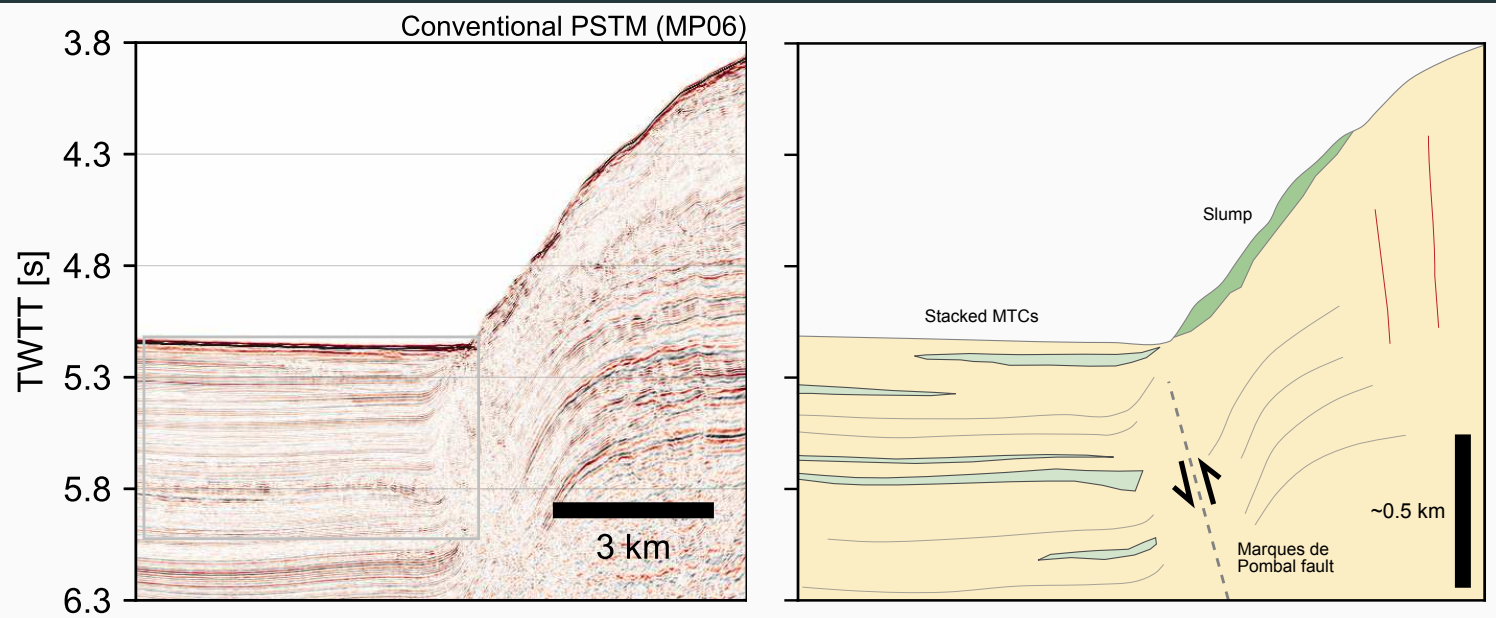


\section{Marques de Pombal Fault}
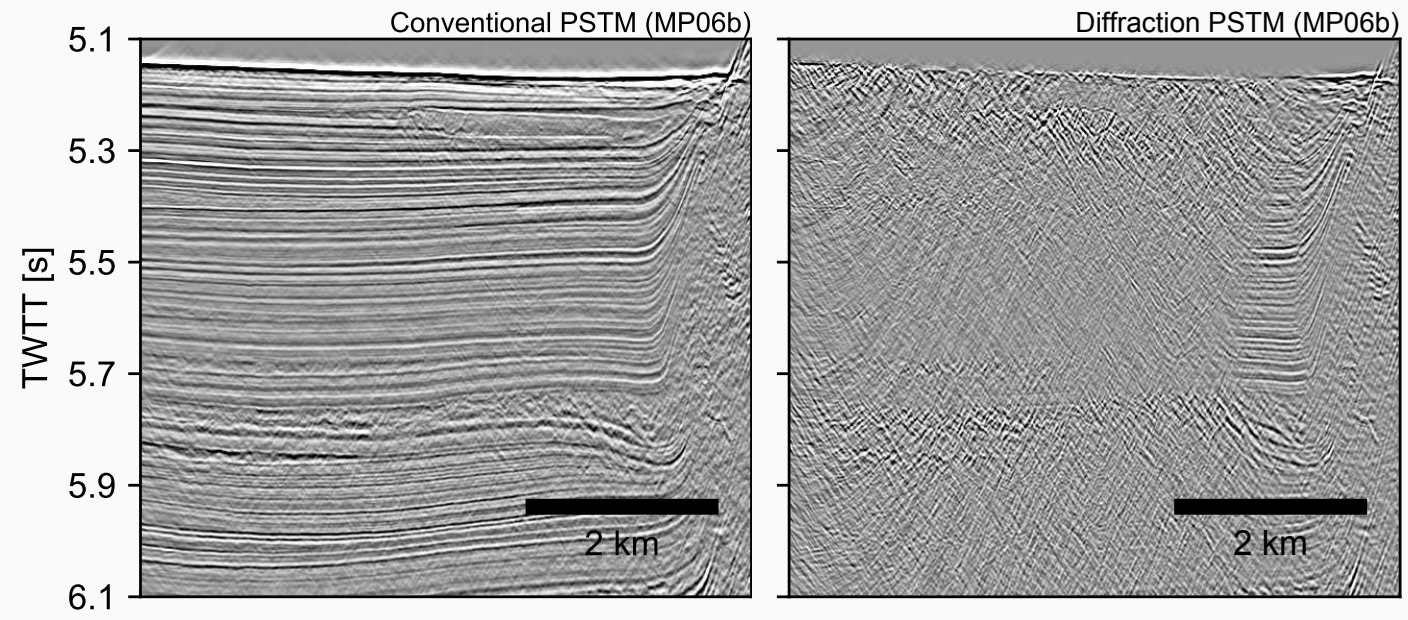

Identify small MTCs (<a wavelet thick), better constraint of lateral extent 


\section{Marques de Pombal Fault}

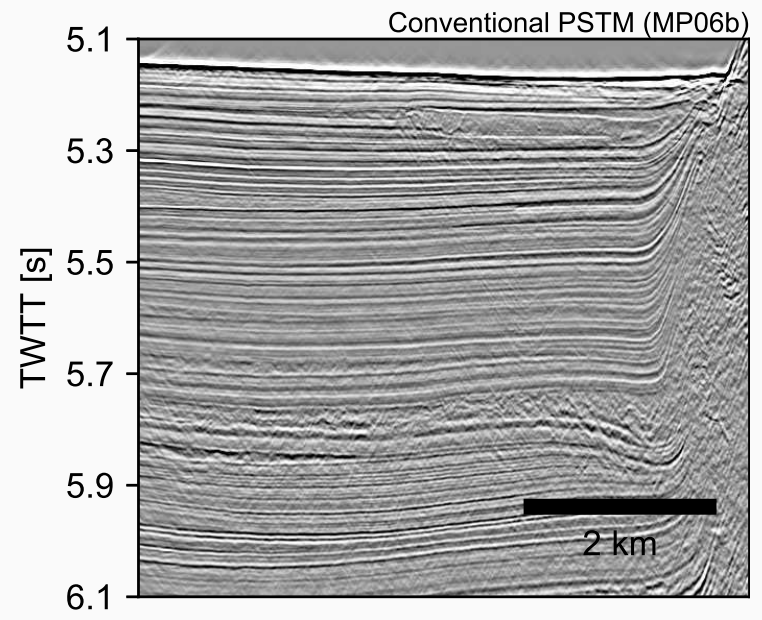

Diffraction energy (MP06b)

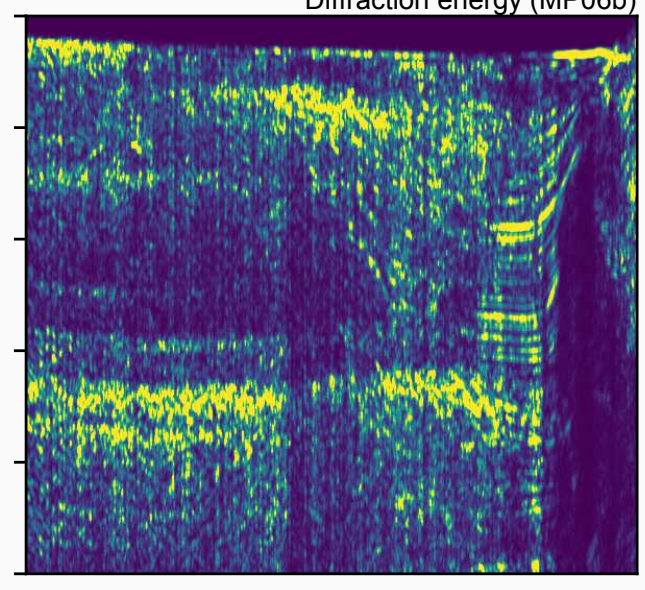

Identify small MTCs (<a wavelet thick), better constraint of lateral extent 


\section{Marques de Pombal Fault}
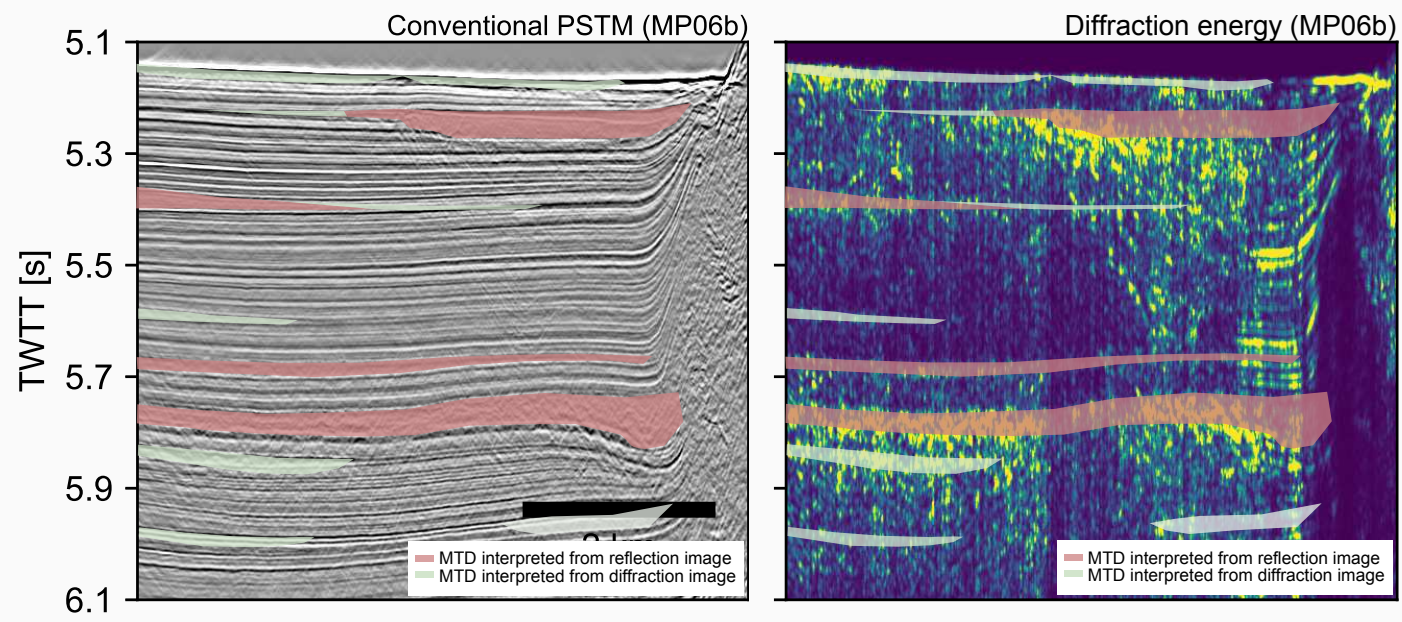

Identify small MTCs (<a wavelet thick), better constraint of lateral extent 
Applications:

- Screening for MTCS, delimiting lateral extent (energy attribute)

- Kinematic indicator from distribution of diffraction energy sensitive to downslope disaggregation of flow?

- Resolve structure inside "transparent" bodies
Limitations:

- Out-of-plane energy on 2-D profiles (3-D effect of MTCS)

- Seismic processing - care needed to preserve diffractions, avoid aliasing

- Noise

Note: works on short-offset and post-stack data (also for velocity model building) 


\section{Conclusions}

1. MTCS do produce lots of diffraction energy, relative to unfailed sediments

2. Diffraction image is sensitive to heterogeneous structure inside MTCS

3. New tool to characterise MTCS

- Image heterogeneous internal structure

- Screen for thin bodies

Conventional PSTM (MP06b)

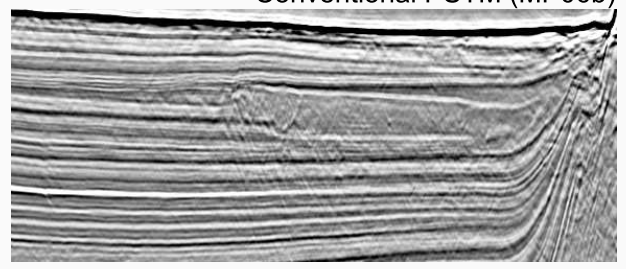

Conventional PSTM (MP06b)

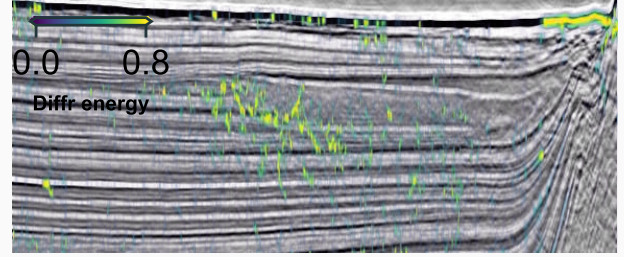




\section{Acknowledgements and references}

Thanks to the crew, technicians and science party of INSIGHT (Leg 1 and 2) cruises, particularly the onboard MCS processing group: E. Piazza, R. Bartolomé, P. Brito and A. Calahorrano

Diffraction separation and imaging was performed using Madagascar (http://reproducibility.org)

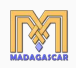

This project has received funding from the European Union's Horizon 2020 research and innovation programme under grant agreement No 721403 .

\section{SLATE "UIII}

This presentation is licensed under a Creative Commons Attribution 4.0 License.

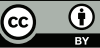

Bull et al., 2009. A review of kinematic indicators from mass-transport complexes using 3D seismic data. Marine and Petroleum Geology.

https://doi.org/10.1016/j.marpetgeo.2008.09.011

Fomel et al., 2007. Poststack velocity analysis by separation and imaging of seismic diffractions. Geophysics.

https://doi.org/10.1190/1.2781533

Yamamoto et al., 2007. Large-scale chaotically mixed sedimentary body within the Late Pliocene to Pleistocene Chikura Group,

Central Japan. Island Arc

https://doi.org/10.1111/j.1440-1738.2007.00587.x

Zitellini et al., 2001. Source of 1755 Lisbon earthquake and tsunami investigated. Eos Trans. AGU.

https://doi.org/10.1029/E0082i026p00285-01 